\title{
Smoking among in-school adolescents in Dar es Salaam, Tanzania: results from the Global Youth Tobacco Survey
}

\author{
ATUPELE KAPITO-TEMBO ${ }^{1}$, ADAMSON S. MUULA ${ }^{1}$, EMMANUEL RUDATSIKIRA ${ }^{2}$ and \\ SETER SIZIYA ${ }^{3}$ \\ ${ }^{1}$ Department of Community Health, University of Malawi, College of Medicine, Blantyre, Malawi \\ ${ }^{2}$ School of Community and Environmental Health, Old Dominion University, Virginia, United States of \\ America \\ ${ }^{3}$ Department of Public Health, University of Zambia, School of Medicine, Lusaka, Zambia
}

\begin{abstract}
Using data from the 2003 Dar es Salaam (Tanzania) Global Youth Tobacco Survey (GYTS), we assessed factors associated with current cigarette smoking among adolescents. We estimated frequencies and conducted logistic regression analysis to identify predictors of current cigarette smoking. Of the 1947 students included in the study, 1893 students indicated their gender of which $53.4 \%$ were females. Most (59.7\%) students were in the 13-15 years age group. Some $13.3 \%$ (95\% CI: $11.8 \%-14.9 \%$ ) reported to have ever tried or experimented with cigarette smoking and 4\% (63/1827) (95\% CI: 2.5-4.2\%) were current cigarette smokers. Factors that were associated with current cigarette smoking were being male, having a friend or parent who smoked, owning an item with a cigarette brand logo, having used other tobacco products other than cigarettes, having some pocket money and having been offered free cigarettes by a tobacco firm representative. Having discussed the harmful effects of smoking with a family member or in school, and exposure to pro-tobacco mass media were not associated with cigarette smoking. Many of the associated factors of cigarette smoking among in-school adolescents in Dar es Salaam are the same as those described previously from the developed nations. However, we found that tobacco-related mass media was not associated with current cigarette smoking. This finding may suggest that the media may not be exerting much influence in facilitating tobacco use in Tanzania.
\end{abstract}

Keywords: adolescent health, cigarette smoking, tobacco, Tanzania

\section{Introduction}

Tobacco is a leading cause of chronic morbidity and deaths across the world. It is estimated that tobacco-associated deaths will increase from about 5.4 million in 2005 to 6.4 million in 2015; tobacco will be responsible for 50\% more deaths than AIDS (Mathers \& Loncar, 2006). Tobacco exposure has effects during prenatal period through childhood and adolescents and well into adulthood (Dejmek et al., 2002; Gilliland et al., 2006; Jain et al., 2005; Key et al., 2007; Mitchell \& Milerad, 2006).

Adolescent cigarette smoking is of great public health significance. Many smokers initiate smoking as adolescents or at least as young adults (Trinidad et al, 2004). Adolescent cigarette smoking has also been reported to be associated with other harmful lifestyles and life experiences such as early initiation of teenage sexual activity, alcohol and illicit drug use and suicide (Lee et al., 2006; Myers \& Kelly, 2006).

At a meeting convened by the World Health Organization's Tobacco Free Initiative (WHO TFI) and the US Centers for Disease Control and Prevention's Office on 
Smoking and Health (CDC OSH) in December 1998, lack of tobacco-related data was identified as a hindrance to the planning and delivery of public health interventions (The GTSS Collaborative Group, 2006a). There are limited data on cigarette smoking among all age groups in Tanzania. The Global Youth Tobacco Survey reported on frequencies and prevalence of smoking and a selected list of variables using the Dar es Salaam GYTS (Global Youth Tobacco Survey, Tanzania, 2003).

Kida et al. (2010) reported the prevalence of tobacco use among adolescents attending secondary school in Temeke district of Dar es Salaam at 23\% and 16\% among boys and girls respectively. Among persons 15 years and older, Jagoe et al. (2002) estimated the prevalence of tobacco smoking is $27 \%$ in males and $5 \%$ in females in Ilala Ilala district, a middle income district in Dar es Salaam.

In order to guide the design, implementation and evaluation of public health interventions aimed to reduce cigarette smoking among adolescents, there is need for reliable data on prevalence and associated factors of smoking among this age group. We used the Dar es Salaam GYTS data to assess these factors associated with smoking among in-school adolescents in Tanzania.

\section{Materials and Methods}

The Dare es Salaam (Tanzania) GYTS conducted in 2003 was a cross sectional study, aimed to recruit in-school adolescents 13 to 15 years old to estimate prevalence of tobacco use. Data were also collected on socio-demographic characteristics, exposure to media, parental smoking, peer smoking and exposure to support that may assist the teenager to stop smoking or not start smoking.

The survey used a two-stage probability sampling technique. In the first stage, primary sampling units were schools which were selected with a probability proportional to their enrolment size. In the second step, a systematic sample of classes in the selected schools was obtained and all students in the selected classes were eligible to participate. A questionnaire was used and included 'core GYTS' and other additional questions as has been described elsewhere regarding the GYTS methodology (The Global Youth Tobacco Survey Surveillance System Collaborating Group, 2005; The GTSS Collaborative Group, 2006a; 2006b). Current cigarette smoking was defined as having smoked even a single puff within the last 30 days preceding the survey. For the purposes of our study however, we aimed to assess factors associated with smoking. These factors were obtained from the literature on tobacco studies (Kyrlesi, 2007; The Global Youth Tobacco Survey Surveillance System Collaborating Group, 2005; The GTSS Collaborative Group, 2006a; 2006b). Data analysis was performed using SAS (version 9.1). We obtained frequencies and their 95\% confidence intervals. We also conducted logistic regression analysis to determine predictors of current cigarette smoking.

All the recruited schools participated i.e. school response rate was 100 percent. Students were recruited from standards 6 and 7 and Forms 1 and 2. The student response rate was $92.5 \%$, thus making the overall response rate was $92.5 \%$. A total of 1947 students participated in the Tanzania - Dar Es Salaam GYTS. 


\section{Results}

\section{Characteristics of the study population}

Of the 1947 students who participated in our study, 1893 students indicated their sex of which $53.4 \%$ were females. Overall $59.7 \%$ of the study population was aged 13 years to 15 years and $22.9 \%$ were 16 years or older.

\section{Lifetime prevalence of cigarette smoking}

Of the 1860 students who indicated whether or not they ever tried or experimented with cigarette smoking, $13.3 \%$ (95\% CI: $11.8 \%-14.9 \%$ ) reported to have ever tried or experimented with cigarette smoking. The overall proportion of students who reported experimenting with smoking increased with increasing age from $11.8 \%$ for those at seven years of age or younger peaking at the age of twelve or 13 years to $26.4 \%$ then started to decline (Figure 1). Some 64.5\% (155/239) of those who reported experimenting with cigarette smoking were boys and generally across all age groups, boys were more likely than girls to report experimenting or trying smoking cigarettes. Some $41.3 \%$ $(65 / 158)$ of the students first tried or experimented with a cigarette when they were eleven years old or younger.

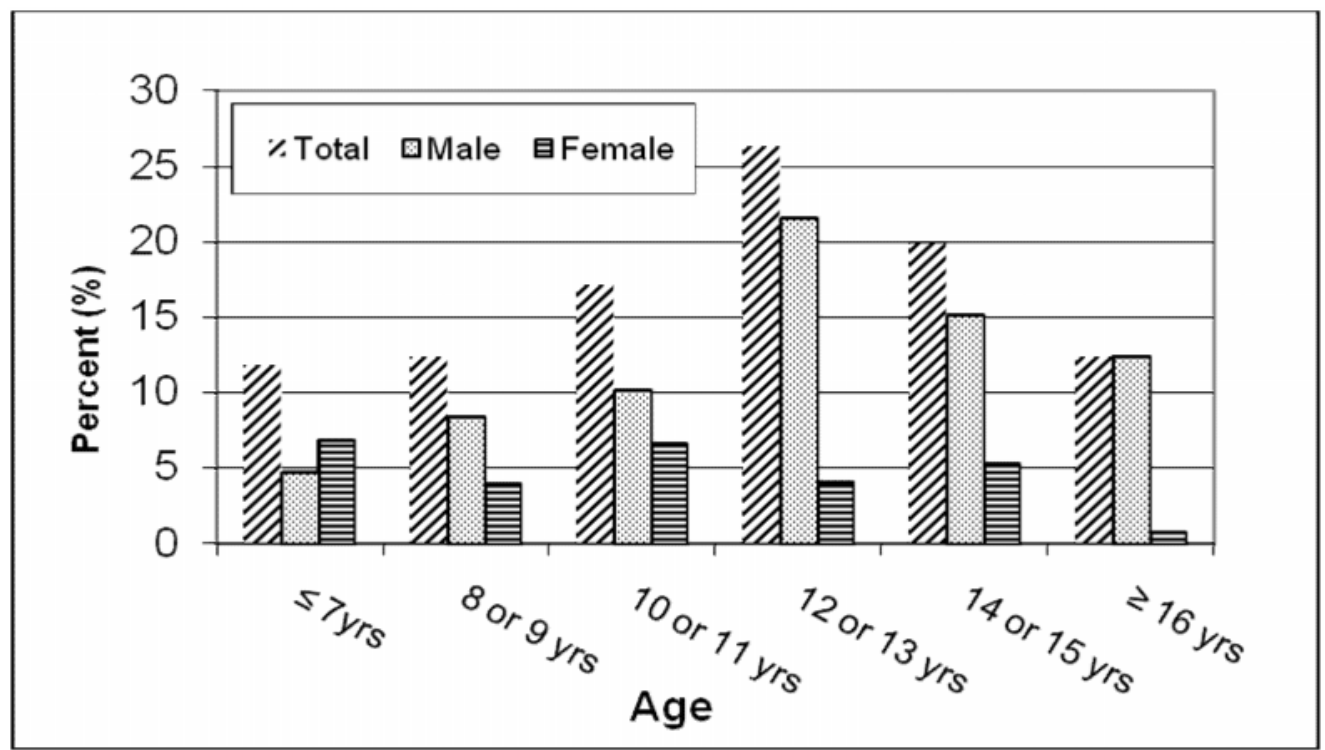

Figure 1: Reported age at initiation of cigarette smoking stratified by gender

\section{Current cigarette smoking and associated factors}

Only 3.4\% (63/1827) (95\% CI: 2.5-4.2\%) of the students reported being current cigarette smokers, defined as smoking cigarette on at least one day during the past 30 days. Male students were 1.8 times more likely to be current smokers compared to female students (Table 1). Students who received a monthly pocket money allowance of less than 1000 Tanzanian Shillings were 4.5 times more likely to be current smokers compared to 
students who reported not receiving any pocket money. Those that received 1000 to 5000 Shillings and more than 6000 Shillings in a month were 8.3 and 10.6 times more likely to smoke cigarettes compared to those that did not receive any money respectively. This remained significant even after adjusting for age, sex, parental smoking status (Table 1). Students who had one parent who smoked were 3.3 times more likely to be current smokers compared to students whose both parents were non-smokers.

Table 1: Unadjusted and adjusted odds ratios (OR) for factors associated with current cigarette smoking among high school students in 2003 in Dar es Salaam

\begin{tabular}{llllll}
\hline Factor & Response & Total & Cigarette smokers & TOR (95\% CI) & †OR (95\% CI) \\
\hline Sex & Female & 966 & 24 & 1 & 1 \\
& Male & 817 & 36 & $1.8(1.1-3.1)$ & $1.7(1.0-3.1)$ \\
Age & $\leq 11$ yrs & 89 & 5 & $2.2(0.8-6.1)$ & $1.6(0.5-5.2)$ \\
& $12-13 y r s$ & 604 & 16 & 1 & 1 \\
& $14-15$ yrs & 702 & 23 & $1.3(0.7-2.4)$ & $1.0(0.5-2.0)$ \\
Monthly & $\geq 16$ yrs & 405 & 17 & $1.7(0.8-3.3)$ & $0.7(0.3-1.5)$ \\
allowance & None & 1396 & 22 & 1 & 1 \\
& $<1000$ & 241 & 16 & $4.5(2.3-8.7)$ & $4.5(2.2-9.3)$ \\
& $1000-5000$ & 97 & 11 & $8.3(3.9-17.8)$ & $7.6(3.1-18.7)$ \\
Parental & $\geq 6000$ & 75 & 11 & $10.6(4.9-22.9)$ & $11.2(5.2-24.0)$ \\
smoking & None & 1520 & 37 & 1 & 1 \\
status & One parent only & 204 & 16 & $3.3(1.8-6.1)$ & $3.4(1.8-6.6)$ \\
& Both & 27 & 4 & $6.6(2.2-20.2)$ & $7.2(2.5-20.7)$ \\
& Don't know & 66 & 4 & $2.6(0.9-7.5)$ & $2.3(0.6-9.1)$ \\
\hline
\end{tabular}

+ Crude OR; $\ddagger=$ Adjusted for age, sex, monthly allowance and parental smoking status

\section{Other tobacco products use}

Despite low prevalence of cigarette smoking among the study population, $7.8 \%$ of the respondents reported using other forms of tobacco like cigars, snuffs, chewing tobacco, pipe, cigarillos or dips. Some $6.8 \%$ (120/1742) of the non-smokers reported using other forms of tobacco compared to $24.5 \%(15 / 61)$ among cigarette smokers ( $p=0.003)$. The use of other tobacco products was not significantly different between the sexes; $8.6 \%$ (75/871) among males versus 7.1\% (71/997) among females.

\section{Access to cigarettes and smoking habits}

Students who were current cigarette smokers reported usually smoking at school, $49.4 \%$ (30/62), 23.8\% (15/62) at home, 12.4\% (8/62) usually smoked either at friends house or in public places like parks, shopping centers or street corners and the rest reported smoking at social events or in other places. While the majority $79.6 \%(50 / 63)$ of current cigarette smokers reported smoking one to five days in the past 30 days, $7.6 \%(5 / 63)$ of them reported smoking daily. Most of the cigarette smokers, 82.5\% (5/63), reported smoking either one or less than a cigarette in a day in the past month. Being given a cigarette by an older person was the most frequent method of getting cigarettes among cigarette smokers as reported by $26.9 \%$ (14/52) followed by $22.9 \%(12 / 52)$ who reported purchasing from a store, shop or vendors while the rest obtained cigarettes through 
some other ways that included borrowing from friends and buying from vending machines. While $33.1 \%(20 / 61)$ of cigarette smokers reported having no friends who smoke, $58.7 \%$ (36/61) of the current smokers reported having some friends who smoke, and $8.3 \%$ reported either having most or all of their friends smoking $(\mathrm{p}<0.05)$.

\section{Exposure to anti-tobacco information and tobacco advertisements}

Overall, $74.4 \%$ of the respondents reported that someone in their family had discussed the harmful effects of smoking with them. However, even though not statistically significant, a smaller proportion of the current cigarette smokers $65.0 \%(39 / 50)$ reported that someone in their family discussed with them the harmful effects of smoking compared to $75.3 \%(1317 / 1754)$ among cigarette non-smokers $(\mathrm{p}=0.07) .51 .3 \%(32 / 62)$ of the cigarette smokers reported ever receiving help or advice from either a friend or program/professional, while $17.6 \%$ reported receiving help from a family member compared to $5.6 \%(100 / 1751)$ and $3.3 \%$ among cigarette non-smokers respectively. Though not statistically significant, a smaller proportion of cigarette smokers $54.4 \%$ (32/59) reported being taught in their classes about dangers of smoking compared to $65.9 \%(1114 / 1711)$ among non-smokers $(\mathrm{p}=0.1)$. There was no significant difference in exposure to anti-smoking media messages (radio, posters, newspapers, magazines, movies) that were seen between cigarette smokers and non-smokers ( $\mathrm{p}=0.08)$. However, $42.6 \%$ of the non-smokers reported seeing anti-smoking messages in public events like concerts, social gatherings, sports events, fairs and community events compared to $34.6 \%(P=0.04)$.

Current cigarette smokers were more likely to have an item with a cigarette logo compared to cigarette none-smokers, OR 2.0 (95\% CI: 1.1-3.5). A greater proportion of cigarette smokers $17.0 \%(10 / 57)$ reported being offered a free cigarette by a cigarette representative compared to $6.6 \%(114 / 1719)$ among cigarette non-smokers $(P=0.002)$. There was no significant difference in exposure to cigarette advertisements between cigarette smokers and non-smokers during public events $(P=0.1)$. However, there was a significant difference in the exposure to cigarette advertisements in newspapers and magazines between cigarette smokers of whom $40.4 \%$ reported seeing advertisements a lot in the past 30 days compared to $35.2 \%$ among cigarette non-smokers $(P=0.01)$.

\section{Discussion}

We estimated that $13.3 \%$ of in-school adolescents in Dar es Salaam had ever tried or experimented with cigarette smoking. Only $4 \%$ of the students were current cigarette smokers. The prevalence of current cigarette smoking in Dar es Salaam is much lower than estimates reported from Arua in Uganda (Mpabulungi \& Muula, 2004, 2006) and Lilongwe in Malawi (Muula \& Mpabulungi (2007). While Uganda has tried to prevent adolescent cigarette smoking (Simpson, 2000), Nsimba and Sussman (2006) have reported that there was no concerted public health intervention aimed to prevent smoking in Tanzania around the time that data for the current study were obtained. In the case of Arua, a rural tobacco growing area of Uganda, a higher prevalence of current 
cigarette smoking could be explained by the tolerance towards tobacco in this tobacco growing area.

Factors that were identified as being associated with current cigarette smoking were being male, having some pocket money, having a friend or parent who smoked, owning an item with a cigarette brand logo, having used other tobacco products other than cigarettes, and having been offered free cigarette by a tobacco firm representative. Having discussed the harmful effects of smoking with a family member or in school and exposure to pro-tobacco mass media were not associated with cigarette smoking. The factors that were identified as associated with cigarette smoking among Tanzanian adolescents were similar to those reported from previous studies in other settings (Bricker et al., 2006; Hoffman et al., 2006; Kyrlesi et al., 2007; Mohan et al., 2005; Parna et al., 2003; Zhang et al., 2005). That current cigarette smokers were also likely to be users of other forms of tobacco probably suggests the clustering of unhealthy behaviours within individuals i.e. adolescents who smoke cigarette may also be likely to engage in other unhealthy behaviours (Faeh et al., 2006).

That many of the smokers smoked at school or at home perhaps suggest that school-based and home-based tobacco intervention programmes could be valuable in Dar es Salaam (Thomas \& Perera, 2006; Thomas et al., 2007). In addition, as smokers were more likely to have friends who were smokers suggest that interventions aimed at reducing adolescent smoking should take into consideration that one's peers are part of the quitting programme.

It is interesting to note that there was no difference between current smokers and non smokers' exposure to multiple mass media anti-tobacco advertisements. This may suggest that these initiatives by tobacco firms may have limited effect on adolescents in Tanzania. In other settings (countries) exposure to mass media tobacco advertisement and owning an item with a tobacco logo were associated with being a current cigarette smoker (Muula et al., 2008; Zulu et al., 2009).

Our study had some important limitations. Questionnaires were self-completed and so the accuracy of the responses was based on memory and willingness to report truthfully (Hung et al., 2006). We did not use biomarkers to ascertain exposure to tobacco smokers by assessing either blood cotinine levels or exhaled carbon monoxide (Jagoe et al., 2002; Jenkins \& Counts, 1999; Low et al., 2004). However other authors have reported that the reliability of adolescents in the United States in providing self report of various behaviours was high (Brener et al., 2001). How far such reliability may be applicable in Tanzania is currently not known. Also recruiting of study participants was restricted to in-school adolescents. To the extent that these in-school adolescents are not similar to out-of-school adolescents, our findings may not be applicable to out of school adolescents. Finally, the data used in the current study were collected eight years ago. However, in the absence of regular tobacco-related data these data and findings could still provide insights as to the problem of cigarette and overall tobacco use.

In conclusion, we have reported the factors that are associated with cigarette smoking among in-school adolescents in Dar es Salaam, Tanzania. We are unaware of any pervious studies that have reported on such factors in this setting. However many 
of the same variables that are associated with cigarette smoking in other settings are also of importance in Tanzania. We suggest that the design, implementation and evaluation of public health interventions aimed to reduce adolescent smoking be made with due considerations to these associated factors.

\section{Competing Interests}

The authors have no conflict of interest to declare.

\section{Acknowledgements}

We thank the World Health Organization and the Centers for Disease Control and Prevention (USA) for making the data available to us for re-analysis.

\section{References}

Brener, N.D., Kann, L., McManus, T., Kinchen, S.A., Sundberg, E.C. \& Ross, J.G. (2002) Reliability of the 1999 Youth Risk Behaviour Survey questionnaire. Journal of Adolescent Health, 31: 336-42.

Bricker, J.B., Peterson, A.V. Jr, Andersen, M.R., Rajan, K.B., Leroux, B.G. \& Sarason, I.G. (2006) Childhood friends who smoke: do they influence adolescents to make smoking transitions? Addiction Behavior, 31: 889-900.

Dejmek, J., Solansky, I., Podrazilova, K. \& Slam, R.J. (2002) The exposure of nonsmoking and smoking mothers to environmental tobacco smoke during different gestational phases and fetal growth. Environmental Health Perspectives, 110: 601-6.

Faeh, D., Viswanathan, B., Chiolero, A., Warren, W. \& Bovet, P. (2006). Clustering of smoking, alcohol drinking and cannabis use in adolescents in a rapidly developing country. BMC Public Health, 6:169.

Gilliland, F.D., Islam, T., Berhane, K., Gauderman, W.J., McConnell, R., Avol, E.\& Peters, J.M. (2006) Regular smoking and asthma incidence in adolescents. American Journal of Respiratory and Critical Care Medicine, 174: 1094-100.

Global Tobacco Surveillance System Collaborating Group (2005) Global Tobacco Surveillance System (GTSS): purpose, production and potential. Journal of School Health, 75: 15-24.

Global Youth Tobacco Survey, Tanzania. (2003). Tanzania-Dar es Salaam Global Youth Tobacco Survey (GYTS) Fact Sheet. Accessed $8^{\text {th }}$ May 2007 from :www.afro.who.int/tfi/publications/gyts_factsheet/tanzania/dar_es_salaam.doc

Gritz, ER, Prokhorov, AV, Hudmon KS, Mullin Jones, M., Rosenblum, C., Chang C.C., Chamberlain, R.M., Taylor, W.C., Johnston, D., \& de Moor, C. (2003) Predictors of susceptibility of smoking and ever-smoking: longitudinal study of a triethnic sample of adolescents. Nicotine and Tobacco Research 5, 493-506. 
Hoffman, B.R, Sussman, S., Unger, J.B. \& Valente, T.W. (2006) Peer influences on adolescent cigarette smoking: a theoretical review of the literature. Substance Use $\mathcal{E}$ Misuse, 41: 103-55.

Hung, J., Lin, C.H., Wang, J.D. \& Chann, C.C. (2006) Exhaled carbon monoxide level as an indicator of cigarette consumption in a workplace cessation program in Taiwan. Journal of Formosan Medical Association 105:210-3

Jagoe, K., Edwards, R., Mugusi, F., Whiting, D. \& Unwin, N. (2002) Tobacco smoking in Tanzania, East Africa: population based smoking prevalence using expired alveolar carbon monoxide as a validation tool. Tobacco Control 11, 210-214.

Jain, R.V., Mills, P.K. \& Parikh-Patel, A. (2005) Cancer incidence in the south Asian population of California, 1988-2000. Journal of Carcinogenesis 4: 21

Jenkins, R.A. \& Counts, R.W. (1999) Personal exposure to environmental tobacco smoke: salivary cotinine, airborne nicotine, and nonsmoker misclassification. Journal of Exposure Analysis and Environmental Epidemiology 9: 352-63.

Key, A.P., Ferguson, M., Molfese, D.L., Paech K, Lehman, C. \& Molfese, V.J. (2007) Smoking during pregnancy affects speech-processing ability in newborn infants. Environmental Health Perspectives, 115: 613-9.

Kida, I.A., Manyori, C. \& Masalu, J.R. (2010) Prevalence and correlates of perceived oral malodor among adolescents in Temeke district, Dar es Salaam. East African Journal of Public Health, 7: 49-53.

Kyrlesi, A., Soteriades E.S., Warren, C.W., Kremastinou, J., Papastergiou, P., Jones, N.R. \& Hadjichristodolou, C. (2007) Tobacco use among students aged 13-15 years in Greece: the GYTS project. BMC Public Health 7: 3.

Lee, L.K., Chen, P.C, Lee, K.K. \& Kaur, J. (2006) Premarital sexual intercourse among adolescents in Malaysia: a cross sectional Malaysian survey. Singapore Medical Journal 47, 476-81.

Low, E.C., Ong, M.C. \& Tan, M. (2004) Breath carbon monoxide as an indication of smoking habit in the military setting. Singapore Medical Journal 45, 578- 582.

Mathers, C.D. \& Loncar D. (2006) Projections of global mortality and burden of disease from 2002 to 2030. PLoS Medicine, 3: e442

Mitchell, E.A. \& Milerad, J. (2006) Smoking and sudden infant death syndrome. Reviews on Environmental Health 21, 81-103.

Mohan, S., Sankara Sarma, P. \& Thankappan K.R. (2005) Access to pocket money and low educational performance predict tobacco use among adolescent boys in Kerala, India. Preventive Medicine 41, 685-692.

Mpabulungi, L. \& Muula, A.S. (2004) Tobacco use among high schools students in Kampala, Uganda: a questionnaire study. Croatia Medical Journal 45, 80-83.

Mpabulungi, L. \& Muula, A.S. (2006) Tobacco use among high school students in a remote district of Arua, Uganda. Rural Remote Health 6: 609.

Muula A.S., Siziya S. \& Rudatsikira, E. (2008) Cigarette smoking and associated factors among in-school adolescents in Jamaica: comparison of the Global Youth Tobacco Surveys 2000 and 2006. BMC Research Notes 5:55. 
Muula, A.S. \& Mpabulungi, L. (2007) Cigarette smoking prevalence among school-going adolescents in two African capital cities: Kampala Uganda and Lilongwe Malawi. African Health Sciences 7: 45-9.

Myers, M.G. \& Kelly, J.E. (2006) Cigarette smoking among adolescents with alcohol and other drug use problems. Alcohol Research and Health 29: 221-7.

Nsimba, S.E.D \& Sussman, S. (2006) Tobacco advertisements and promotion industry on smoking in Tanzania: a review of negative public health implications for current and future generations. Tobacco Induced Diseases 3: 41-43.

Parna, K., Rahu, K., Fischer, K., Mussalo-Rauhamaa, H., Zhuravleva, I., Umbleja, T. \& Rahu, M. (2003) Smoking and associated factors among adolescents in Tallinn, Helsinki and Moscow: a multilevel analysis. Scandinavian Journal of Public Health, 31, 350-358.

Simpson, D. (2000) Uganda athlete's fight BAT's abuse of sports. Tobacco Control 9: 129130.

The GTSS Collaborative Group (2006a) The Global Tobacco Surveillance System. Tobacco Control 15: 1-3.

The GTSS Collaborative Group (2006b). A cross country comparison of exposure to secondhand smoke among youth. Tobacco Control 15: 4-19.

Thomas, R. \& Perera, R. (2006) School-based programmes for preventing smoking. Cochrane Database Systematic Reviews, 3, CD001293.

Thomas, R., Baker, P., \& Lorenzetti D. (2007) Family-based programs for preventing smoking by children and adolescents. Cochrane Database Systematic Reviews 1, CD004493.

Trinidad, D.R., Gilpin, E.A., Lie, L., \& Pierce, J.P. (2004) Do the majority of AsianAmericans and African-American smokers start as adults? American Journal of Preventive Medicine 26, 156-158.

Zhang, L., Wang, W.F., \& Zhou, G. (2005) A cross-sectional study of smoking risk factors in junior high school students in Henan, China. Southeast Asian Journal of Tropical Medicine and Public Health 36, 1580-1584.

Zulu, R., Siziya, S., Muula, A.S. \& Rudatsikira, E. (2009). Association of advertisementpromotion-sponsorship with current cigarette smoking among in-school adolescents in Zambia. Annals of African Medicine 8, 229-235. 